\title{
Smart Age Detection for Social Media
}

\author{
Using Deep Learning Techniques via Ear Shape
}

\author{
Manal Alghieth ${ }^{1}$, Jawaher Alhuthail ${ }^{2}$, Kholod Aldhubiay ${ }^{3}$, Rotan Alshowaye ${ }^{4}$ \\ Information Technology, Qassim University, Qassim, Saudi Arabia
}

\begin{abstract}
Over the recent years, there has been an immense attraction towards age detection due to its raised implementation in various sectors. Such as government regulations and rules, security control, and human computer interaction. Popular human features such as the face and fingerprints can be modified or changed with time. However, ear has a stable structure that does not change with time and have unique features that satisfies the requirements of a biometric trait. This research presents a detailed analysis extracting the features of the human ear only by applying Deep Learning techniques. In particular, Convolutional Neural Network (CNN) is applied on large datasets which have multiple layers to extract the features and classify them. The proposed methodology increased the number of the dataset by collecting more private children datasets, and consequently achieved high accuracy by $98.75 \%$ along with amending the architecture of the selected neural network compared to previous studies. This research can be benefited to control the contents of social media by detecting the age of group whether it is under 18 or above 18.
\end{abstract}

Keywords-Age detection; deep learning; ear features; CNN; control social media

\section{INTRODUCTION}

The rapid development of technology and science has benefited humanity in different fields. Simultaneously, there has been increasing interest in age estimation.

Ear is rich in unique features and it is one of the most usable forms of authentication. Among various biometric traits, ear is a stable structure that does not change with age and satisfies the requirements of a biometric trait. This gives it an advantage compared with other popular human features such as the face and fingerprints, which can change with time or can be modified. Ear images can be analyzed using deep learning techniques to detect age of a person.

The main idea of deep learning is that, a different type of data can be implemented into a machine to learn certain features which can lead to an excellent decision making for the classification. Deep Learning is considered as a new field of machine learning, it has achieved great results and high accuracy in various areas compared to human experts such as image classification which takes an input and predict a particular class, Object detection, Image retrieval, Semantic segmentation and Human pose estimation. Deep learning can be divided into four categories as shown in Fig. 1. The most appealing characteristic to use deep learning, the fact that it can be applied on large datasets, the increasing ability of processing, the more layers a network has the higher the level of features it will learn. One of the most powerful techniques in deep learning is neural networks that contains numerous amounts of neurons which offers answers to different kinds of problems. Neural network is a combination of algorithms that is applied in identifying various patterns, like numerical, images, audio, text or time series. The NN types are: Convolutional Neural Networks, Recursive Neural Networks, Unsupervised Pre-trained Networks and Recurrent Neural Networks [1].

The Convolutional Neural Networks (CNN) is an outstanding deep learning algorithm. It consists of three layers: the convolutional layer, the pooling layer and the fullyconnected layers. It can be applied to large dataset like images and videos to extract features and classify it. CNN require much less preprocessing compared to other algorithms. Recent articles have proved that $\mathrm{CNN}$ is highly effective and already exceeded the accuracy of human experts [2].

Many techniques have been developed to authenticate and verify the identity of humans and age detection.

For instance, Decision tree J48 classifier applied on FG_NET. It consists of three phases image preprocessing, data mining, estimation and evaluation [3].

Deep CNN model called VGG-Face. It contains 11 layers, eight convolutional layers and 3 fully connected layers that were applied on a database for face recognition task. It was modified and fine-tuned to perform age estimation on the Adience database [4].

Convolutional Neural Networks architectures were applied to classify ear appearance-based features. These architectures include AlexNet, VGG, GoogLeNet and SqueezeNet [5].

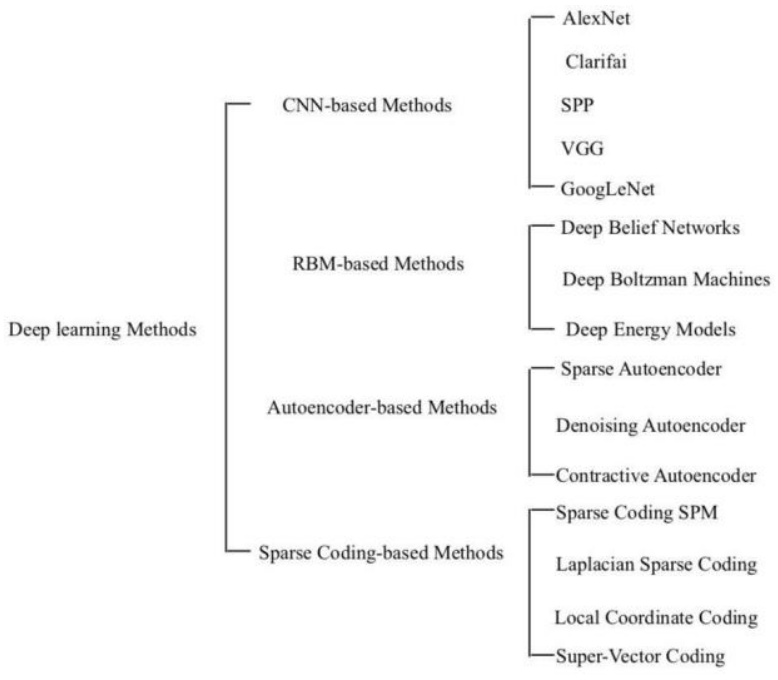

Fig. 1. Deep Learning Categories. 
Other techniques were applied on ear geometric features. Such as, Logistic regression provides a linear discrimination between different classes. Its objective is to minimize the logistic function. Random Forests is an ensemble machine learning algorithm and was used for classification as well as feature selection. It works with sub-trees that are learned on a part of the training data that uses different samples from training set instead of using all of them and the average result is calculated from different sub-trees. Support vector machine is a supervised machine learning algorithm which can be used for both classification and regression. It is based on the theory of statistical learning [5].

\section{LITERATURE REVIEW}

A few attempts have been done on detecting the age via the ear shape. For instance, a detailed analysis on identifying soft biometric traits, age and gender, from ear images was presented. In [5], it uses geometric features and appearancebased features to illustrate the ear morphology. These features have been classified by employing four different methods: logistic regression, random forests, support vector machines, and neural networks. The gender classification resulted in accuracy of $94 \%$ and for the age classification achieved a $52 \%$ accuracy. Furthermore, the work in [6] focused on using profile face images and ear images as input to build a multimodal deep neural network for age and gender classification in order to enhance the accuracy. It trained their model on the UND-F, UND-J2, and FERET datasets for gender classification and on the FERET dataset for age classification. The experiment indicated that profile face images have numerous amounts of features that can support age and gender classification and merging profile face and ear image can reach great accuracies. Compared to the reported age classification accuracy in the previous work, the study has increased the accuracy by $9 \%$ for five class age group classification on FERET dataset and reached $60.97 \%$ of accuracy.

To support the proposed methodology, studies about ear features have proved ear is a unique body part that can provide numerous information. M. Taura, L. Adamu, A. Gudaji, and M. Modibbo [7], discussed the possibility to determine age from ear shape by testing the correlation between age and ear length, width and index for both left and right sides. The study applied the regression equation for age detection. It proved the application of external ear morphometry that can serve as additional tool for age estimation of individuals. The human ear is stable and does not change over time and that have been presented. In [8],[9] and [10], it has been proven through studies that the shape of ear of the same person show some similarities but are not strictly symmetric. Ear verification can also be applied to differentiate between identical twins, due to its unique features; there are no two ears that have the same helix.

On the other hand, face images can be used to detect age where in research [4], Z. Qawaqneh, A. A. Mallouh, and B. D. Barkana, proposed a model to detect age using deep CNN model called VGG-Face that was applied on a database for face recognition task. It was modified and fine-tuned to perform age estimation on the Audience database. A trained
CNN model for face recognition was used in this research for age detection and resulted in great outcomes. This work proved that employing a pre-trained $\mathrm{CNN}$ on large data set can overcome over fitting issues. The proposed model achieved a higher accuracy by $9 \%$. The work in [11] applied three methods on facial images to detect age: Feature Extraction , Hierarchical age estimation and Age Estimate Fusion. The research proved that using face image can be employed effectively in various areas to detect age. In [3], it discussed the potential of determining age from facial images using machine learning technique, and applied J48 classifier on FG_NET dataset. The experiment proved that the proposed system can give results with high precision and low time complexity, using decision tree J48 classifier resulted in high precision rate with low time taken on the FG_NET dataset with accuracy of $89.13 \%$ and time taken is 0.023 .

Recently, there has been a growing interest in age estimation, due to its raised implementation in various sectors such as government regulations and rules, security control and human computer interaction. In spite of the advances in age estimation, it is still a challenge. There are two main problems in detecting the age by ear shape, lack of ear dataset collection and age classification accuracy. One of the previous studies [5] have reached 52\% accuracy and they claim that it is because of the short number of datasets. Their theory states that the more datasets used the more accuracy they will get. Hence, this research will attempt to improve accuracy by using deep learning techniques. It requires a large dataset to give more accuracy and can also extract ear features by itself. There are several ways applied on ear features to detect the age, such as logistic regression, random forest, support vector machines and neural network.

The popular human features such as face and fingerprints can be modified or changed with time. However, ear has unique features and it is a stable structure that does not change with time and satisfies the requirements of a biometric trait. Nowadays, users of social media can give false information without validation, hence users can explore any content available, whether it is age appropriate or not. This research contributes in controlling the content of the watched media depending on the user's age via the ear shape only.

\section{PRoposed Methodology}

This research proposes a system that detect the age by applying ear dataset into deep learning algorithms which will analyze the images and extract the features via multiple hidden layers of deep learning and classify them. The model was trained on AMI, AWE, and a Private Children dataset. The resulted classification will give the system the output needed which is the age group whether above 18 or under 18 . The proposed model is shown in Fig. 2.

The implementation begins with collecting ear images from different sources (This model used 3000 ear images which was divided into $80 \%$ for training and $20 \%$ for testing) and using csv files to handle the data. Importing the necessary python libraries for deep learning, load and process the data, then defining the architecture of CNN model and how to apply it. Fig. 3 shows the algorithm used in this research. 


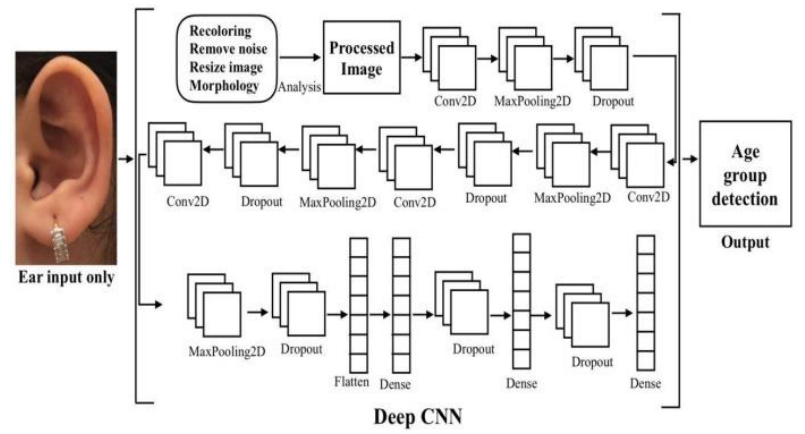

Fig. 2. Proposed Methodology Model.

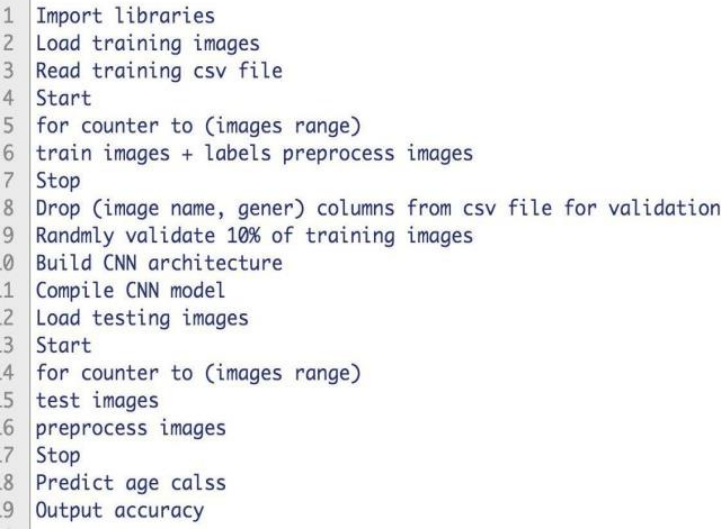

Fig. 3. Smart Age Detection Algorithm.

The images were different in sizes and shape for that a preprocessing step was applied before the training and testing.

Training the model using the training images set and their corresponding true labels, then it will pass the validation images to assure the performance of the model. Also, in this phase the number of epochs is defined.
The architecture of the applied model includes deciding the number of hidden layers, number of neurons in each layer and what activation function embedded in each layer to convert an input signal to an output signal, to be use the output signal as an input in the next layer. For the 2D convolution layer it uses many filters in a single layer that learns ear features, the extracted features used as an input to the next layer. Following conv2D, a max pooling layer was performed to reduce the amount of pixels computation that needs to be done and sends the most important data to the next layer. The utilized hidden layers are Dropout (drops random unit of CNN model and to prevent overfitting) and Flattened (flattens all the features maps into a single column). Lastly, the fully connected layer (Dense layer) is the output layer that will classify the ear images either above 18 or under 18 years. This research contributes in applying two activation functions in different layers, ReLU (Rectified Linear Unit) and Sigmoid were applied. ReLU activation function was embedded in each layer; except the output layer a Sigmoid activation function was performed to predict the probability as an output, which is excellent for the binary classification task.

For testing, loading $20 \%$ of the testing images set with testing csv file without labels. Before making predictions, the model will preprocess these images using the same steps mentioned in the training phase. Then it will predict the age for these ears of human using the trained model. Finally, the model will classify the tested images either more than 18 or less than 18 years old.

\section{RESULTS}

The major findings were classifying images to detect the age either above 18 or under 18 for social media and the exceptional accuracy of $98.75 \%$. Table I shows the result related to previous studies and proven the higher accuracy that has been achieved in this research.

TABLE. I. COMPARISON BETWEen the Proposed Method AND PREVIOUS STUdies

\begin{tabular}{|c|c|c|c|c|c|}
\hline Title & Dataset & Overall Accuracy & Method & Year & Age Group \\
\hline $\begin{array}{l}\text { The Prediction of Old and Young Subjects } \\
\text { from Iris Texture[12] }\end{array}$ & 100 Subjects & $64 \%$ & $\begin{array}{l}\text { Rando-mForest } \\
\text { Algorit-hm }\end{array}$ & 2013 & $\begin{array}{l}\text { Young: (22-25), } \\
\text { Old: }(35+)\end{array}$ \\
\hline $\begin{array}{l}\text { Deep Convolutional Neural Network for } \\
\text { Age Estimation based on } \\
\text { VGG-Face Model[4] }\end{array}$ & Adience database & $59.9 \%$ & Deep CNN & 2017 & $\begin{array}{l}0-2,4-6,8-13,15- \\
20,25-32,38-43 \\
48-53,60+\end{array}$ \\
\hline $\begin{array}{l}\text { Intelligent Age Estimation From Facial } \\
\text { Images Using Machine Learning } \\
\text { Techniques[3] }\end{array}$ & FG_NET dataset & $89.13 \%$ & $\begin{array}{l}\text { Decisio-n tree J48 } \\
\text { classifie-r Algorit-hm }\end{array}$ & 2018 & $\begin{array}{l}\text { Class1: }(3-7,26-30), \\
\text { Class2: }(8-25), \\
\text { Class3: }(31-50)\end{array}$ \\
\hline $\begin{array}{l}\text { Age and Gender Classification from Ear } \\
\text { Images [5] }\end{array}$ & UND J ear Database & $52 \%$ & Deep CNN & 2018 & $\begin{array}{l}18-28,29-38,39- \\
48,49-58,59-68+\end{array}$ \\
\hline $\begin{array}{l}\text { Multimodal Age and Gender Classification } \\
\text { Using Ear and Profile Face Images[6] }\end{array}$ & FERET & $60.97 \%$ & Deep CNN & 2019 & $\begin{array}{l}18-28,29-38,39- \\
48,49-58,59-68+\end{array}$ \\
\hline $\begin{array}{l}\text { Smart Age Detection For Social Media } \\
\text { Using Deep Learning Techniques via Ear } \\
\text { Shape } \\
\text { (The proposed method) }\end{array}$ & $\begin{array}{l}\text { AMI, AWE \& Private } \\
\text { Children datasets }\end{array}$ & $98.75 \%$ & Deep CNN & 2019 & $\begin{array}{l}\text { Above } 18 \\
\& \\
\text { Under } 18\end{array}$ \\
\hline
\end{tabular}




\section{DISCUSSION}

CNN is one of deep learning architectures and it was applied in this research to classify the ear images. The lack of classified dataset limited the research from identifying age in different kinds of groups such as child, teenagers, adult or elderly. However, above 18 or under 18 age group can be extremely useful in applying this to the social media and the most important thing is to identify adults or young generation, that can allow controlling the appropriate watched content. In the future utilizing the medical aspect can help in improving the extraction of more features and producing better accuracy result for different group of ages. This research used the shape of ear only that contains unique features and the chosen architecture succeeded and resulted in a great accuracy. A collaboration with biologist could be made to perform a better accuracy based on the features of the ear itself.

\section{CONCLUSION}

This research proposed a model to identify human age either above 18 or under 18 based on ear shape only by using deep CNN model. It applied most appropriate activation function to make the model more accurate. This study contributed in applying two activation functions, ReLU which was embedded in each layer except the output layer and Sigmoid activation function was performed to predict the probability as an output.

The proposed model reached an exceptional accuracy of $98.75 \%$. Due to the high accuracy of detecting the age groups, this research can be applied in social media platforms to authenticate user's information.

\section{REFERENCES}

[1] Y. Guo, Y. Liu, A. Oerlemans, S. Lao, S. Wu, and M. S. Lew, "Deep learning for visual understanding: A review," Neurocomputing, vol. 187, pp. 27-48, 2016.

[2] J. Gu et al., "Recent advances in convolutional neural networks," Pattern Recognit., vol. 77, pp. 354-377, 2018.

[3] A. R. Abbas, "Intelligent Age Estimation From Facial Images Using Machine Learning Techniques," Iraqi J. Sci., vol. 59, no. 2A, 2018.

[4] Z. Qawaqneh, A. A. Mallouh, and B. D. Barkana, "Deep Convolutional Neural Network for Age Estimation based on VGG-Face Model," no. 1, 2017.

[5] D. Yaman, F. I. Eyiokur, N. Sezgin, and H. K. Ekenel, "Age and gender classification from ear images," IWBF 2018 - Proc. 2018 6th Int. Work. Biometrics Forensics, pp. 1-7, 2018.

[6] D. Yaman and F. I. Eyiokur, "Multimodal Age and Gender Classification Using Ear and Profile Face Images."

[7] M. Taura, L. Adamu, A. Gudaji, and M. Modibbo, "Application of external ear morphometry in age prediction: a pilot study," Int. J. Res. Med. Sci., vol. 3, no. 7, pp. 1775-1779, 2015.

[8] S. Dodge, J. Mounsef, and L. Karam, "Unconstrained ear recognition using deep neural networks," IET Biometrics, 2018.

[9] A. Sabet, K. Kamal, A. Ghany, and H. Elmahdy, "Human Ear Recognition Using Geometrical Features Extraction," Procedia Procedia Comput. Sci., vol. 65, no. Iccmit, pp. 529-537, 2015.

[10] I. Omara, F. Li, H. Zhang, and W. Zuo, "A novel geometric feature extraction method for ear recognition," Expert Syst. Appl., vol. 65, pp. 127-135, 2016.

[11] S. E. Padme and P. S. Desai, "Estimation of Age from Face Images," Int. J. Sci. Res., vol. 4, no. 12, pp. 1927-1931, 2016.

[12] A. Sgroi, K. W. Bowyer, and P. J. Flynn, "The prediction of old and young subjects from iris texture," Proc. - 2013 Int. Conf. Biometrics, ICB 2013, pp. 1-5, 2013. 